\title{
Car Sickness
}

National Cancer Institute

\section{Source}

National Cancer Institute. Car Sickness. NCI Thesaurus. Code C34451.

A sensation of discomfort associated with ground travel; symptoms may include nausea, vomiting, dizziness or sweating. 